\title{
Integração Espacial do Mercado de Boi Gordo: uma Análise de Cointegração com Threshold
}

\section{Spatial Integration in the Brazilian Beef Cattle Market: an Analysis with Thresholds Cointegration}

\author{
Dênis Antônio da Cunha* \\ João Eustáquio de Lima** \\ Marcelo José Braga***
}

Resumo: Os estudos tradicionais de integração de mercados, baseados na metodologia de cointegração, admitem que não haja descontinuidades no processo de transmissão de preços entre localidades espacialmente separadas. Todavia, a existência de custos de transação pode criar assimetrias ou mesmo impedir o processo. Dessa forma, este trabalho tem o objetivo principal de realizar um estudo de integração do mercado brasileiro de boi gordo, levando em conta esse aspecto. Utilizaram-se modelos de cointegração com threshold (TAR e M-TAR). Na análise empírica foram considerados os principais estados brasileiros produtores de boi gordo, no período de 1994 a 2008. Os resultados indicaram que os mercados são integrados, mas os custos de transação influenciam a ligação espacial entre eles, já que os choques negativos de baixa magnitude são eliminados de maneira distinta que os positivos. Nesses casos certamente os benefícios advindos de alterações nos preços seriam menores que os custos de ajuste.

Palavras-chave: Integração de mercados. Cointegração com threshold. Custos de transação. Boi gordo.

Abstract: The traditional studies of market integration, based on the cointegration methodology, consider that there are no discontinuities in the process of price transmission between spatially separated localities. However, the existence of transaction costs may create asymmetries or even block the process of integration. The main objective of this paper was to analyze the possible effects of transaction costs in the price adjustment mechanism of market integration of beef cattle in Brazil using models of cointegration with threshold, namely, TAR and M-TAR. It

\footnotetext{
* Doutorando pelo Programa de Pós-Graduação em Economia Aplicada do Departamento de Economia Rural da Universidade Federal de Viçosa. E-mail: denis.cunha@ufv.br

** Doutor em Economia Rural, Professor Titular do Departamento de Economia Rural da Universidade Federal de Viçosa. E-mail: jelima@ufv.br

*** Doutor em Economia Aplicada, Professor Associado do Departamento de Economia Rural da Universidade Federal de Viçosa. E-mail: mjbraga@ufv.br
} 
was used data on prices received by producers in the most important states during the period of august of 1994 through august 2008. Results indicated state market integration but with significant effect of transaction costs since negative shocks or shocks of low magnitude are eliminated distinctly from positive shocks. In these cases the benefits for transaction coming from prices variations are lower than the adjustment costs resulting in impediment of the market integration process.

Keywords: Market integration. Threshold cointegration. Transaction costs. Beef cattle.

JEL Classification: C50; D23; D40.

\section{Introdução}

Ao longo do tempo, um grande número de estudos tem procurado analisar a integração entre mercados agropecuários. Faminow e Benson (1990) consideraram que localidades espacialmente separadas e que comercializam um mesmo produto formariam um mercado integrado se os seus preços fossem determinados de maneira interdependente, ou seja, alterações de preços numa região seriam transmitidas às demais.

Segundo Goletti e Christina-Tsigas (1995), análises dessa natureza permitem inferir sobre alocação de recursos e orientar e garantir maior efetividade das políticas de previsão e estabilização de preços. Ademais, a decisão sobre a infraestrutura de comercialização mais adequada para o desenvolvimento agrícola do país pode ser facilitada pelo conhecimento dos fatores estruturais responsáveis pela integração.

Os métodos utilizados nas análises de integração foram se modificando paralelamente ao desenvolvimento das técnicas econométricas. O reconhecimento de que os preços, em geral, são variáveis não estacionárias estimulou, a partir da década de 1990, uma extensa literatura que utiliza testes de cointegração para inferir a respeito das relações espaciais entre os mercados.

A ideia intuitiva de cointegração, derivada dos estudos de Engle e Granger (1987) e Johansen (1988), é que variáveis não estacionárias podem apresentar trajetórias temporais interligadas, de modo que no longo prazo tenham uma relação de equilíbrio. Como resultado, a existência de cointegração é um indício de interdependência dos preços num mercado, podendo-se afirmar que se um conjunto de preços for cointegrado, choques de oferta ou demanda que surgirem numa localidade seriam transferidos às demais.

Segundo Mattos (2008), a técnica tradicional de cointegração pressupõe que em mercados integrados quaisquer variações de preços numa localidade sempre serão transmitidas a outro(s) mercado(s), ainda que os ganhos obtidos com o ajuste sejam inferiores aos custos incorridos. No 
entanto, a existência de custos relacionados com movimentação de bens e informações entre os mercados restringe a transmissão de choques de preços, fazendo com que o processo se dê de maneira descontínua ou simplesmente não ocorra.

Desde meados da década de 1990, alguns estudos têm sido desenvolvidos com o intuito de levar em consideração os custos de transação e, dessa forma, expressar de modo mais apropriado a relação entre os mercados. Podem ser citados os trabalhos de Obstfeld e Taylor (1997), Goodwin e Piggott (2001) e Lo e Zivot (2001). No Brasil, estudos dessa natureza ainda são raros, podendo-se destacar os de Mattos e Lima (2006) e Mattos (2008).

Mesmo utilizando apenas dados de preços nesses estudos, já que os custos de transação são de difícil mensurabilidade, pressupõe-se a existência de um ajustamento assimétrico, ou seja, a presença de não linearidades nas relações entre os mercados, o que é feito por meio de um modelo de cointegração com threshold. De acordo com Goodwin e Holt (1999), considera-se que choques de grande magnitude, acima de certo limite (threshold), induzam ajustamentos em magnitudes distintas daqueles decorrentes de choques abaixo do threshold, em razão dos custos relacionados com alteração dos preços.

Diante do exposto, este trabalho tem o objetivo principal de realizar um estudo de integração que considere a possibilidade de assimetrias na transmissão de preços, resultantes da existência de custos de transação. O mercado brasileiro de boi gordo foi escolhido para a análise empírica devido à sua importância para a economia nacional e também por tratar-se de uma commodity cujo processo de comercialização envolve elevados custos, devido à dificuldade de transportar os animais e de a carne, por ser um produto perecível, requerer cuidados especiais no armazenamento e transporte.

De fato, os custos de transação têm sido uma preocupação dos agentes que atuam no setor. Segundo Urso (2007), para reduzir os altos custos associados à compra e ao transporte de animais, desde meados da década de 1990, os frigoríficos têm se estabelecido próximos às localidades produtoras. Com esse expediente, as firmas diminuem custos com fretes, pagamentos de impostos (como ICMS) ${ }^{1}$, entre outros. Os pecuaristas, por sua vez, passaram a comercializar seus animais principalmente com as empresas localizadas nas proximidades da sua região.

$1 \quad$ ICMS é a sigla que identifica o Imposto sobre Operações relativas à Circulação de Mercadorias e sobre Prestações de Serviços de Transporte Interestadual e Intermunicipal e de Comunicação. É um imposto que cada um dos Estados e o Distrito Federal podem instituir, como determina a Constituição Federal de 1988. 
A pecuária de corte brasileira ocupa papel de destaque tanto nacional quanto internacionalmente. Conforme dados do Conselho Nacional da Pecuária de Corte (2007), o Brasil é o segundo maior produtor e, desde 2004, é o principal exportador mundial de carne bovina. Na Tabela 1, é apresentada a evolução de alguns indicadores econômicos do setor. Destaca-se o grande consumo per capita que fomenta a comercialização interna, que é uma condição necessária para a integração dos mercados.

Tabela 1 - Indicadores Econômicos da Pecuária Brasileira no Período de 1994 a 2007 (anos selecionados)

\begin{tabular}{c|c|c|c|c|c|c|c|c}
\hline & 1994 & 1996 & 1998 & 2000 & 2002 & 2004 & 2006 & 2007 \\
\hline Rebanho bovino (milhões) & 158.2 & 153.1 & 157.8 & 164.3 & 179.2 & 192.5 & 204.7 & 207.2 \\
\hline Taxa de abate (\%) & 16.43 & 20.25 & 19.14 & 19.8 & 19.82 & 21.51 & 21.67 & 21.72 \\
\hline Consumo per capita (kg) & 32.6 & 38.0 & 35.3 & 36.3 & 36.6 & 36.4 & 36.6 & 36.7 \\
\hline $\begin{array}{c}\text { Produção / carne } \\
\text { (mil toneladas) }\end{array}$ & 5.20 & 6.05 & 6.04 & 6.65 & 7.30 & 8.35 & 8.95 & 9.20 \\
\hline Exportação (mil toneladas) & 0.37 & 0.27 & 0.37 & 0.59 & 1.01 & 1.85 & 2.20 & 2.35 \\
\hline Exportação (US\$ bilhões) & 0.57 & 0.44 & 0.58 & 0.78 & 1.10 & 2.45 & 3.80 & 4.50 \\
\hline
\end{tabular}

Fonte: CONSELHO NACIONAL DA PECUÁRIA DE CORTE, 2007.

Deve-se mencionar, no entanto, que a integração do mercado brasileiro de boi gordo é uma questão que já foi analisada por vários autores. Zen (1997) verificou a existência de transmissão de preços entre as diversas praças produtoras na região centro-sul do país e seus resultados evidenciaram um mercado altamente integrado, com respostas rápidas a alterações de preços em cada uma das localidades. O estudo de Pereira (2005) focou a integração em nível estadual e também indicou integração com "grande dinamismo no comportamento dos preços". Urso (2007) analisou as principais praças, em termos de produção e comercialização, e concluiu que São Paulo lidera o processo de formação de preços no mercado brasileiro.

Diferentemente dos estudos citados que utilizaram a técnica tradicional de cointegração, Mattos e Lima (2006) investigaram os efeitos dos custos de transação sobre a integração do mercado de boi gordo, entre os estados de Minas Gerais e São Paulo, no período de 1972 a 2005. A conclusão foi que o ajustamento de preços a eventuais choques ocorria de maneira diferenciada, dependendo da magnitude do desvio em relação ao equilíbrio de longo prazo, o que sugere que os custos de transação entre os mercados estudados eram significativos.

Portanto, pretende-se levar em conta os efeitos de custos de transação no estudo da integração do mercado brasileiro de boi gordo. Serão considerados os principais estados produtores, o que possibilitará uma 
análise mais abrangente da integração, já que, conforme González-Rivera e Helfand (2001), os mercados apresentam natureza multilocacional. Além disso, considerando várias regiões produtoras (com infraestrutura de transporte e comunicação distintas), pode-se também verificar se há diferenças no processo de transmissão de preços entre localidades geograficamente mais distantes entre si, uma vez que a distância tente a elevar os diversos custos associados à comercialização. ${ }^{2}$

Deseja-se avançar no entendimento dos efeitos dos custos de transação sobre a integração do mercado de boi gordo utilizando metodologia de cointegração com threshold que não apenas identifica assimetrias no processo de transmissão de preços. Em outras palavras, busca-se identificar se a assimetria é caracterizada por velocidade de ajuste de preços distinta quanto a choques positivos e negativos que surgem no mercado. ${ }^{3}$ Esse tipo de análise é importante pois há discussão na literatura a respeito de que os aumentos de preços numa região tendem a ser transmitidos às diversas localidades que formam um mercado integrado mais rapidamente do que as quedas.

Por fim, o horizonte temporal considerado na análise compreende os anos de 1994 a 2008. Esse período foi escolhido porque foi a partir de meados da década de 1990 que o setor começou a passar pelas mudanças que objetivavam reduzir os custos de transação. Dessa forma, pode-se avaliar se tais alterações tiveram efeito sobre o valor do threshold, comparando os resultados do presente estudo aos de Mattos e Lima (2006).

Além desta introdução, o trabalho está dividido em outras três seções. Na segunda, são apresentados os aspectos teóricos relacionados com integração de mercados, o modelo analítico utilizado, bem como a descrição e a fonte dos dados; na terceira, os resultados obtidos e, na quarta, as principais conclusões.

\section{Metodologia}

\subsection{Integração de Mercados: Conceitos e Modelo Analíitico}

Segundo Fackler e Goodwin (2001), a integração de mercados referese à interdependência dos preços em diferentes regiões, ao longo do tempo.

2 Essa também é uma sugestão de pesquisa de Mattos e Lima (2006). Segundo esses autores, deve-se analisar outros estados brasileiros, sobretudo aqueles mais distantes de São Paulo e Minas Gerais, para os quais os custos de transação e seus efeitos sobre a integração devem ser ainda maiores.

3 O modelo econométrico que permite tal análise é o Momentum-Threshold (M-TAR), que será descrito na seção 2 . 
Por compartilharem informações de longo prazo semelhantes, os preços de um mercado integrado sofrerão influências não apenas das condições de oferta e demanda locais, mas também das condições das demais localidades. Dessa forma, a integração pode ser vista como uma medida do grau em que os choques de demanda e oferta que surgem numa região são transmitidos a outra.

O relacionamento entre os preços em localidades espacialmente separadas e que comercializam entre si depende do tamanho dos custos de transação. De acordo com González-Rivera e Helfand (2003), no contexto de integração de mercados, tais custos incluem comissões relacionadas com procura e transferência de bens, despesas originadas da negociação e imposição contratual, custos financeiros, taxas, custos de transporte e o custo de oportunidade do tempo alocado na procura por informações.

Segundo a condição de arbitragem espacial, se houver diferença entre os preços, os arbitradores comprarão o produto no mercado de preço mais baixo e o venderão onde for mais caro, até que a diferença máxima seja o custo relacionado com transferência de mercadorias de uma localidade a outra. Alternativamente, poder-se-á falar em preços autárquicos, se a diferença não for grande o suficiente para que os lucros potenciais excedam os custos de transação (FAMINOW; BENSON, 1990). Um processo eficiente de arbitragem conduzirá à estabilidade do preço no mercado.

Dessa forma, os custos de transação limitam o "espaço" da arbitragem, fazendo com que os participantes do mercado prefiram transacionar com localidades geograficamente mais próximas, o que afeta a integração de mercado e a eficiência dela resultante. Portanto, tais custos desempenham papel fundamental nesse tipo de análise e não deveriam ser ignorados.

A análise tradicional de integração de mercados, baseada no teste de cointegração bivariado de Engle e Granger (1987), consiste em ajustar a seguinte equação:

$$
P_{j, t}=\beta_{0}+\beta_{1} P_{i, t}+\mu_{t}
$$

em que $P_{\mathrm{j}, t}$ e $P_{\mathrm{i}, \mathrm{t}}$ são os preços praticados nos mercados $j$ e $i$, respectivamente e $\mu_{t}$, termo de erro aleatório. Para que haja integração, a combinação linear entre $P_{j, t}$ e $P_{i, t}$, ou seja, $\mu_{\mathrm{t}}=P_{\mathrm{j}, \mathrm{t}}-\beta_{0}-\beta_{1} P_{\mathrm{i}, \mathrm{t}}$, deve ser estacionária. A análise objetiva verificar a estacionariedade de $\mu_{t}$ por meio de um teste de raiz unitária do tipo $\mathrm{ADF}$ (Augmented Dickey-Fuller): ${ }^{4}$

$4 \quad$ Por ser amplamente utilizado e conhecido na literatura de séries temporais, os detalhes do desenvolvimento do teste $\mathrm{ADF}$ foram omitidos. Uma descrição completa pode ser obtida em Enders (1995), capítulo 4, p. 221-238. Contudo, deve-se observar que, como $\mu_{t}$ é uma variável estimada, os valores críticos do teste não seguem a distribuição original tabulada por Dickey e Fuller (1979). Os valores críticos adequados ao teste podem ser obtidos em Engle e Granger (1987) ou MacKinnon (1991). 


$$
\Delta \hat{\mu}_{t}=\rho \hat{\mu}_{t-1}+\gamma_{i} \sum_{i=1}^{p-1} \Delta \hat{\mu}_{t-i}+\varepsilon_{t}
$$

Se a hipótese nula de não estacionariedade (presença de raiz unitária) for rejeitada $(\rho=0)$, conclui-se pela cointegração entre as séries de preços e, consequentemente, pela integração dos mercados $i$ e j. Entretanto, se $P_{\mathrm{j}, \mathrm{t}}+\tau_{\mathrm{ji}}>P_{\mathrm{i}, \mathrm{t}}$ em que $\tau_{\mathrm{ji}}$ é o custo de transação relacionado com transferência de produtos de $j$ para $i$, não haverá incentivo à ação dos arbitradores e, portanto, os mercados não serão integrados, ainda que os preços sejam cointegrados.

Uma forma de contornar essa limitação do método de Engle e Granger (1987), para a análise de integração de mercados, é especificar um modelo que leve em conta que a diferença entre $P_{j, t} e P_{i, t}$ possa ser menor que os custos de transação e, por isso, impedir o ajuste de preços (ou restringir a choques de determinada magnitude). Por conseguinte, a cointegração com threshold é a metodologia mais adequada para analisar a integração de mercados, quando existir a possibilidade de que haja assimetrias na transmissão de preços.

Como os custos de transação não são considerados explicitamente, eles passam a fazer parte do termo de erro de (1). Dessa forma, o método utilizado neste estudo, conforme proposto por Enders e Granger (1998) e Enders e Siklos (2001), consiste em especificar $\mu_{t}$ como um modelo autorregressivo com threshold (TAR) $:^{5}$

$$
\Delta \hat{\mu}_{t}=\rho_{1} I_{t} \hat{\mu}_{t-1}+\rho_{2}\left(1-I_{t}\right) \hat{\mu}_{t-1}+\varepsilon_{t}
$$

em que $\hat{\mu}_{t}$ é a série de resíduos obtidos de (1); $I_{t}$, variável dummy que terá o valor 1 , se $\hat{\mu}_{t-1} \geq \tau$, e 0 , se $\hat{\mu}_{t-1}<\tau$, em que $\tau$, que representa $o$ parâmetro threshold, divide o comportamento da série; $\varepsilon_{t}$, termo de erro, independente de $\hat{\mu}_{t}$ e $\tau$, idd $\sim\left(0, \sigma^{2}\right)$. Os valores de $\rho_{1}$ e $\rho_{2}$ captam o ajustamento assimétrico, ou seja, se $\hat{\mu}_{\mathrm{t}-1}$ for positivo, o ajuste será dado por $\rho_{1} \hat{\mu}_{t-1}$, mas, se for negativo, será captado por $\rho_{2} \hat{\mu}_{t-1}$.

A análise pode ser refinada se for considerado que o ajustamento exibe uma trajetória mais prolongada em determinada direção. Conforme Enders e Granger (1998), pode-se verificar, por exemplo, se um choque positivo tende a persistir, enquanto um negativo tende a reverter-se rapidamente ao threshold. Nesse caso, o TAR representado em (3), é reescrito na forma de um momentum-threshold (M-TAR):

5 Assim como o teste $\mathrm{ADF}$, se houver autocorrelação serial nos resíduos, o modelo poderá ser reescrito na forma "aumentada": $\Delta \hat{\mu}_{t}=\rho_{1} I_{t} \hat{\mu}_{t-1}+\rho_{2}\left(1-I_{t}\right) \hat{\mu}_{t-1}+\gamma_{i} \sum_{i=1}^{p-1} \Delta \hat{\mu}_{t-i}+\varepsilon_{t}$. Por tratar- se de uma série de resíduos, o modelo não deverá conter intercepto e tendência.

CUNHA, D. A.; LIMA, J. E.; BRAGA, M. J. Integração espacial do mercado brasileiro de boi gordo... 


$$
\Delta \hat{\mu}_{t}=\rho_{1} M_{t} \hat{\mu}_{t-1}+\rho_{2}\left(1-M_{t}\right) \hat{\mu}_{t-1}+\varepsilon_{t}
$$

em que $M_{t}$ é uma dummy que terá valor 1 , se $\Delta \hat{\mu}_{t-1} \geq \tau$ e 0 , se $\Delta \hat{\mu}_{t-1}<\tau$. Segundo Ewing, Hammoudeh e Thompson (2006), o modelo M-TAR pressupõe que $\hat{\mu}_{t}$ exiba um momentum maior numa direção do que na outra; o ajustamento será modelado por $\rho_{1} \hat{\mu}_{t-1}$, se $\Delta \hat{\mu}_{t}$ estiver acima do threshold e por $\rho_{2} \hat{\mu}_{\mathrm{t}-1}$, se $\Delta \hat{\mu}_{t}$ estiver abaixo.

$\mathrm{O}$ valor do parâmetro threshold $(\tau)$ é, a priori, desconhecido. Sua correta estimação pode ser realizada pelo método descrito por Chan (1993). A metodologia consiste em ordenar os resíduos estimados em (1), em ordem crescente, e excluir os 15\% menores e os 15\% maiores valores. Deve-se, posteriormente, estimar o modelo escolhido (TAR e/ou M-TAR) utilizando cada um dos $70 \%$ valores de $\hat{\mu}_{t}$ restantes. $O$ estimador consistente do threshold $(\hat{\tau})$ será aquele cuja equação apresentar a menor soma de quadrados dos resíduos. Deve-se destacar que, se $\hat{\tau}=0$, ter-se-á o modelo convencional de Engle e Granger (1987) e não haverá assimetrias na transmissão de preços.

Considerando que, para choques de preços abaixo de certo limite (threshold), os benefícios advindos do ajustamento poderiam não cobrir os custos relacionados com alteração dos preços, a estimativa do parâmetro $\hat{\tau}$ poderá ser entendida como uma aproximação dos custos de transação existentes.

A análise da integração do mercado consiste em testar, tanto em (3) quanto em (4), a hipótese nula de não cointegração, ou seja, $\rho_{1}=\rho_{2}=0$. Devem-se utilizar as estatísticas $\Phi$ (para o caso do modelo TAR) ou $\Phi^{m}$ (M-TAR), tabuladas por Enders e Siklos (2001) ou Wane, Gilbert e Dibooglu (2004). Se a hipótese de não cointegração for rejeitada, testar-se-á uma hipótese de ajustamento simétrico, $\rho_{1}=\rho_{2}$, utilizando o teste $F$ convencional.

\subsection{Dados}

Foram utilizadas séries de preços mensais da arroba de boi gordo, recebidos pelos produtores de São Paulo (SP), Minas Gerais (MG), Espírito Santo (ES), Rio de Janeiro (RJ), Paraná (PR), Santa Catarina (SC), Rio 
Grande do Sul (RS), Mato Grosso (MT), Mato Grosso do Sul (MS) e Goiás (GO), no período de agosto de 1994 a agosto de 2008, disponibilizados pela Fundação Getúlio Vargas (FGV-DADOS). As séries foram deflacionadas pelo IGP-DI (base agosto de 2008) e, posteriormente, logaritmizadas.

Os dez estados, representantes das regiões Sul, Sudeste e CentroOeste do país, responderam, em 2007, por cerca de 65\% do rebanho bovino brasileiro e por $70 \%$ da produção de carne bovina (ANUALPEC, 2008). Embora haja outros estados com expressiva produção, como Pará, Rondônia, Bahia e Tocantins, a indisponibilidade de séries completas de preços para o período de análise foi um fator limitante de sua inclusão.

\section{Resultados da Análise Empírica}

Como primeiro passo da análise de cointegração, foi verificada a estacionariedade das séries de preços. Como a periodicidade das séries é mensal e, em geral, há sazonalidade nos preços de boi gordo, foi utilizado o teste HEGY, que testa a presença de raízes sazonais e não sazonais, conforme apresentado em Madalla e Kim (1998). Na especificação do teste foi incluído um termo de intercepto e as defasagens foram escolhidas por meio do critério de Schwartz (Tabela 2).

A análise da significância estatística de $\pi_{1}$ indicou presença de raiz unitária não sazonal em todas as séries, ou seja, os preços de boi gordo recebidos pelos produtores, nos estados analisados, foram não estacionários (integradas de ordem um, I[1]), ${ }^{6}$ mas as hipóteses de raiz unitária sazonal foram rejeitadas.

Confirmada a não estacionariedade das séries, estimaram-se as relações de cointegração. Para determinação das relações de longo prazo estabelecidas entre os preços dos estados produtores de boi gordo da região Centro-Sul do país, São Paulo foi escolhido como o mercado central. Assim como em Pereira (2005) e Urso (2007), a escolha deste estado deve-se a múltiplos fatores, quais sejam, ele é o principal consumidor de carne bovina do país; é um dos maiores produtores; concentra grande número de frigoríficos além de ser a sede da Bolsa de Mercadorias $\mathcal{E}$ Futuros (BMEZF), que comercializa contratos futuros de boi gordo, sendo dessa forma importante para a formação de preços dessa commodity.

6 Para confirmação da ordem de integração das séries foi realizado um teste ADF em sua primeira diferença. A hipótese nula de raiz unitária foi rejeitada para todos os preços. 


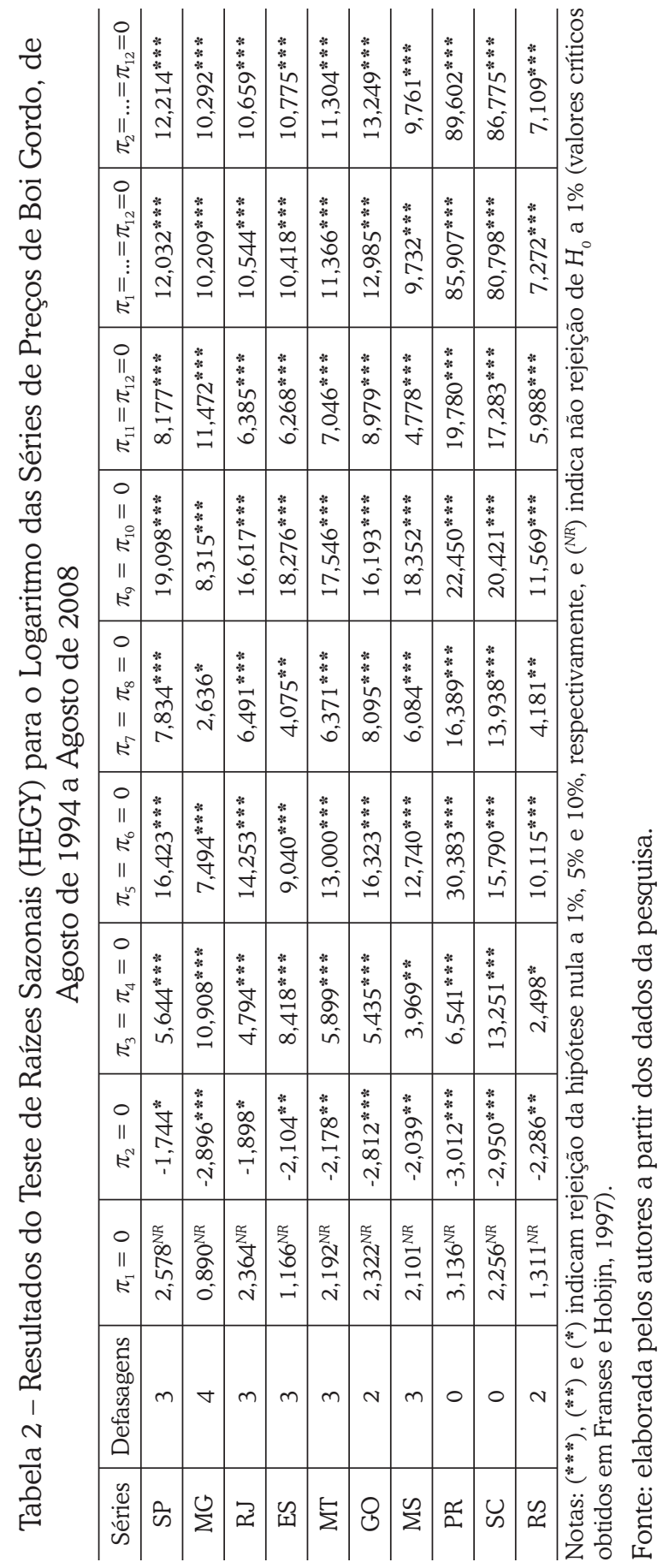


Na Tabela 3 são apresentadas as equações de cointegração, estimadas por MQO, entre os preços de São Paulo e os dos demais estados considerados na análise, segundo o método de Engle e Granger (1987).

Os coeficientes relativos à elasticidade de transmissão de preço de longo prazo foram estatisticamente significativos a $1 \%$ de significância. ${ }^{7}$ As elasticidades são diretamente interpretadas; uma variação de $10 \%$ no preço de São Paulo causaria uma mudança, no mesmo sentido, de 9,3\% no preço de Minas Gerais, de 6,9\% no preço do Rio de Janeiro e assim sucessivamente.

Em geral, mercados com grandes rebanhos bovinos e mais próximos de São Paulo, como Minas Gerais e Mato Grosso do Sul, apresentaram coeficientes de elasticidades mais elevados (0,93 e 0,88, respectivamente) que estados mais distantes, como Rio Grande do Sul e Santa Catarina (0,73 e 0,70). Portanto, como discutido por Mattos (2008), pode-se inferir que há indícios de uma relação inversa entre o grau de transmissão de preços e a distância física entre os mercados.

Tabela 3 - Relações de Cointegração entre o Preço de São Paulo e os Mercados de MG, RJ, ES, MT, GO, MS, PR, SC e RS. Agosto de 1994 a Agosto de 2008

\begin{tabular}{l|rl}
\hline \multicolumn{1}{c|}{ Mercados } & \multicolumn{2}{|c}{ Equação de cointegração $P_{i, t}=\beta_{0}+\beta_{1} P_{S P, t}+\mu_{t}$} \\
\hline São Paulo e Minas Gerais & $M G_{t}=\begin{array}{r}0,2494+0,9254 S P_{t} \\
(0,1817)(0,0420)\end{array}$ & $\begin{array}{l}R^{2}=0,94 \\
D W=1,93\end{array}$ \\
\hline São Paulo e Rio de Janeiro & $R J_{t}=\begin{array}{r}1,2402+0,6925 S P_{t} \\
(0,1735)(0,0393)\end{array}$ & $\begin{array}{l}R^{2}=0,96 \\
D W=2,15\end{array}$ \\
\hline São Paulo e Espírito Santo & $E S_{t}=\begin{array}{r}1,0705+0,7221 S P_{t} \\
(0,1845)(0,0421)\end{array}$ & $\begin{array}{l}R^{2}=0,95 \\
D W=2,08\end{array}$ \\
\hline São Paulo e Mato Grosso & $M T_{t}=\begin{array}{r}0,4684+0,8618 S P_{t} \\
(0,1208)(0,0280)\end{array}$ & $\begin{array}{l}R^{2}=0,97 \\
D W=1,94\end{array}$ \\
\hline São Paulo e Goiás & $G O_{t}=\begin{array}{r}0,4951+0,8659 S P_{t} \\
(0,1377)(0,0318)\end{array}$ & $\begin{array}{l}R^{2}=0,96 \\
D W=2,08\end{array}$ \\
\hline São Paulo e Mato Grosso do Sul & $M S_{t}=\begin{array}{r}0,4718+0,8791 S P_{t} \\
(0,1172)(0,0271)\end{array}$ & $\begin{array}{l}R^{2}=0,95 \\
D W=2,06\end{array}$ \\
\hline São Paulo e Paraná & $P R_{t}=\begin{array}{r}0,6890+0,8280 S P_{t} \\
(0,1233)(0,0285)\end{array}$ & $\begin{array}{l}R^{2}=0,95 \\
D W=2,10\end{array}$ \\
\hline São Paulo e Santa Catarina & $S C_{t}=\begin{aligned} 1,2724+0,7008 S P_{t} \\
(0,2165)(0,0499)\end{aligned}$ & $\begin{array}{l}R^{2}=0,93 \\
D W=1,94\end{array}$ \\
\hline São Paulo e Rio Grande do Sul & $R S_{t}=\begin{aligned} 1,0946+0,7328 S P_{t} \\
(0,2466)(0,0570)\end{aligned}$ & $\begin{array}{l}R^{2}=0,88 \\
D W=1,89\end{array}$ \\
\hline
\end{tabular}

Nota: Os valores entre parênteses referem-se ao erro-padrão do parâmetro estimado; DW refere-se à estatística de Durbin-Watson após a correção de correlação serial dos resíduos.

Fonte: Elaborada pelos autores a partir dos dados da pesquisa.

7 Nesse caso, as inferências baseadas na estatística t-Student somente serão válidas se as séries forem cointegradas. 
O passo seguinte na análise consistiu em utilizar os resíduos de cada relação de cointegração para verificar se, de fato, alterações no preço de São Paulo são transmitidas aos demais mercados. Mas, diferentemente do método de Engle e Granger (1987), por meio do qual se deve realizar um teste ADF nos resíduos, foram estimados os modelos TAR e M-TAR, conforme a metodologia de Enders e Granger (1998) e Enders e Siklos (2001). Os resultados são apresentados na Tabela 4.

O primeiro passo para análise dos resultados obtidos com os modelos TAR e M-TAR foi testar se $\rho_{1}=\rho_{2}=0$, ou seja, ausência de cointegração entre as séries de preços. Para o Rio Grande do Sul (M-TAR), essa hipótese não pôde ser rejeitada nem a 10\% de significância; para o Mato Grosso do Sul (M-TAR), rejeitou-se a 10\% e para os demais pares de mercado, em ambos os modelos, a hipótese foi rejeitada a 1\% de significância, o que confirma que há um equilíbrio de longo prazo entre os preços em estudo. É possível afirmar, portanto, que os estados analisados, exceto o Rio Grande do Sul, formam um mercado integrado.

Em seguida, foi verificado se o ajustamento é assimétrico e, dessa forma, a pertinência de utilização dos modelos que consideram o efeito threshold, em oposição à análise tradicional de Engle e Granger (1987). À exceção do Mato Grosso do Sul (no modelo M-TAR), nos demais mercados a hipótese nula de ajustamento simétrico $\left(\rho_{1}=\rho_{2}\right)$ foi rejeitada a $1 \%$ de significância (no caso de Santa Catarina, para o M-TAR, considerou-se 5\%). Dessa forma, a especificação não linear foi adequada, o que confirma a importância do modelo que considera assimetrias na transmissão de preços.

Tabela 4 - Parâmetros Estimados para os Modelos TAR e M-TAR

\begin{tabular}{|c|c|c|c|c|c|}
\hline Mercados & $\hat{\tau}$ & $\rho_{1}$ & $\rho_{2}$ & $\rho_{1}=\rho_{2}=0$ & $\rho_{1}=\rho_{2}$ \\
\hline \multicolumn{6}{|c|}{ Modelo TAR } \\
\hline São Paulo e Minas Gerais & 0,0003 & $\begin{array}{c}0,1278 * * * \\
(0,0159)\end{array}$ & $\begin{array}{c}-0,0398^{* * *} \\
(0,0197)\end{array}$ & $\begin{array}{c}34,0713^{* * *} \\
{[9,29]}\end{array}$ & $\begin{array}{c}43,3820 * * * \\
\{0,0000\}\end{array}$ \\
\hline São Paulo e Rio de Janeiro & 0,0045 & $\begin{array}{c}0,1585^{* * *} \\
(0,0184)\end{array}$ & $\begin{array}{l}-0,0389^{N S} \\
(0,0267)\end{array}$ & $\begin{array}{c}39,0818^{* * *} \\
{[9,02]}\end{array}$ & $\begin{array}{c}39,2621 * * * \\
\{0,0000\}\end{array}$ \\
\hline São Paulo e Espírito Santo & 0,0026 & $\begin{array}{c}0,5980^{* * *} \\
(0,0622)\end{array}$ & $\begin{array}{c}-0,8398^{* * *} \\
(0,1070)\end{array}$ & $\begin{array}{c}83,7621^{* * *} \\
{[9,29]}\end{array}$ & $\begin{array}{c}145,2767^{* * *} \\
\{0,0000\}\end{array}$ \\
\hline São Paulo e Mato Grosso & 0,0035 & $\begin{array}{c}0,1008 * * * \\
(0,0223) \\
\end{array}$ & $\begin{array}{c}-0,0299^{N S} \\
(0,0224) \\
\end{array}$ & $\begin{array}{c}12,0898^{* * *} \\
{[8,98]}\end{array}$ & $\begin{array}{c}19,6814^{* * *} \\
\{0,0000\} \\
\end{array}$ \\
\hline São Paulo e Goiás & 0,0043 & $\begin{array}{c}4,0727^{* * *} \\
(0,3699)\end{array}$ & $\begin{array}{c}-0,2367^{N S} \\
(0,2907)\end{array}$ & $\begin{array}{c}61,9184^{* * *} \\
{[9,29]}\end{array}$ & $\begin{array}{c}90,2501 * * * \\
\{0,0000\}\end{array}$ \\
\hline $\begin{array}{l}\text { São Paulo } \\
\text { e Mato Grosso do Sul }\end{array}$ & $-0,0064$ & $\begin{array}{c}0,0647 * * * \\
(0,0086)\end{array}$ & $\begin{array}{c}-0,0575^{* * *} \\
(0,0110)\end{array}$ & $\begin{array}{c}41,8947 * * \\
{[9,02]}\end{array}$ & $\begin{array}{c}76,6443 * * * \\
\{0,0000\}\end{array}$ \\
\hline São Paulo e Paraná & $-0,0070$ & $\begin{array}{c}0,0681^{* * *} \\
(0,0063)\end{array}$ & $\begin{array}{c}-0,0713^{* * *} \\
(0,0064)\end{array}$ & $\begin{array}{c}120,3032^{* * *} \\
{[9,29]}\end{array}$ & $\begin{array}{c}240,6058^{* * *} \\
\{0,0000\}\end{array}$ \\
\hline
\end{tabular}


(continuação)

\begin{tabular}{|c|c|c|c|c|c|}
\hline São Paulo e Santa Catarina & $-0,0037$ & $\begin{array}{c}0,2141^{* * *} \\
(0,0321)\end{array}$ & $\begin{array}{c}-0,2516^{N S} \\
(0,1660)\end{array}$ & $\begin{array}{c}22,9748^{* * *} \\
{[8,98]}\end{array}$ & $\begin{array}{l}7,4546^{* * *} \\
\{0,0070\}\end{array}$ \\
\hline $\begin{array}{l}\text { São Paulo } \\
\text { e Rio Grande do Sul }\end{array}$ & 0,0045 & $\begin{array}{c}0,3897 * * * \\
(0,0764) \\
\end{array}$ & $\begin{array}{c}-0,8794 * * * \\
(0,1635) \\
\end{array}$ & $\begin{array}{c}27,0054^{* * *} \\
{[9,29]} \\
\end{array}$ & $\begin{array}{c}48,8074^{* * *} \\
\{0,0000\} \\
\end{array}$ \\
\hline \multicolumn{6}{|c|}{ Modelo M-TAR } \\
\hline São Paulo e Minas Gerais & 0,0346 & $\begin{array}{c}0,1583^{* * * *} \\
(0,0173)\end{array}$ & $\begin{array}{c}-0,0243^{N S} \\
(0,0159) \\
\end{array}$ & $\begin{array}{c}42,9990^{* * *} \\
{[10,40]}\end{array}$ & $\begin{array}{c}59,9494 * * * \\
\{0,0000\}\end{array}$ \\
\hline São Paulo e Rio de Janeiro & 0,0124 & $\begin{array}{c}0,2988^{* * *} \\
(0,0450)\end{array}$ & $\begin{array}{c}-0,3186^{* * *} \\
(0,0796)\end{array}$ & $\begin{array}{c}51,7132 * * * \\
{[10,06]}\end{array}$ & $\begin{array}{c}73,1128^{* * *} \\
\{0,0000\}\end{array}$ \\
\hline São Paulo e Espírito Santo & 0,0326 & $\begin{array}{c}0,2874 * * * \\
(0,0377)\end{array}$ & $\begin{array}{c}-0,0094^{N S} \\
(0,0317)\end{array}$ & $\begin{array}{c}31,2504^{* * *} \\
{[10,40]}\end{array}$ & $\begin{array}{c}46,7693^{* * *} \\
\{0,0000\} \\
\end{array}$ \\
\hline São Paulo e Mato Grosso & $-0,0122$ & $\begin{array}{c}0,0933^{* * *} \\
(0,0226)\end{array}$ & $\begin{array}{c}-0,0128^{N S} \\
(0,0285)\end{array}$ & $\begin{array}{c}10,1283^{* * *} \\
{[10,06]}\end{array}$ & $\begin{array}{c}12,1060 * * * \\
\{0,0006\}\end{array}$ \\
\hline São Paulo e Goiás & 0,0135 & $\begin{array}{c}27,398^{* * * *} \\
(0,3218) \\
\end{array}$ & $\begin{array}{r}0,8338^{* *} \\
(0,3719) \\
\end{array}$ & $\begin{array}{c}36,2546^{* * *} \\
{[10,40]}\end{array}$ & $\begin{array}{c}20,6028^{* * *} \\
\{0,0000\}\end{array}$ \\
\hline $\begin{array}{l}\text { São Paulo } \\
\text { e Mato Grosso do Sul }\end{array}$ & $-0,0312$ & $\begin{array}{c}0,0408^{* * *} \\
(0,0116)\end{array}$ & $\begin{array}{r}0,0635^{* *} \\
(0,0304)\end{array}$ & $\begin{array}{c}6,7111^{*} \\
{[6,58]}\end{array}$ & $\begin{array}{l}0,6170^{N S} \\
\{0,4333\}\end{array}$ \\
\hline São Paulo e Paraná & $-0,0235$ & $\begin{array}{c}0,0532 * * * \\
(0,0088)\end{array}$ & $\begin{array}{c}-0,0482 * * * \\
(0,0155)\end{array}$ & $\begin{array}{c}23,9638^{* * *} \\
{[10,09]}\end{array}$ & $\begin{array}{c}33,5937^{* * *} \\
\{0,0000\} \\
\end{array}$ \\
\hline São Paulo e Santa Catarina & 0,0376 & $\begin{array}{c}0,1386^{* * *} \\
(0,0092)\end{array}$ & $\begin{array}{c}-0,0350^{* * * *} \\
(0,0063)\end{array}$ & $\begin{array}{c}127,9528^{* * *} \\
{[10,40]}\end{array}$ & $\begin{array}{c}241,8209^{* * *} \\
\{0,0000\}\end{array}$ \\
\hline $\begin{array}{l}\text { São Paulo } \\
\text { e Rio Grande do Sul }\end{array}$ & 0,0468 & $\begin{array}{c}0,1602 * * * \\
(0,0512)\end{array}$ & $\begin{array}{l}0,0033^{\text {NS }} \\
(0,0781)\end{array}$ & $\begin{array}{c}6,4248^{\mathrm{NS}} \\
{[6,75]}\end{array}$ & - \\
\hline
\end{tabular}

Notas: $(* *),(* *)$ e $(*)$ indicam significativo a $1 \%, 5 \%$ e $10 \%$; $\left({ }^{\text {NS }}\right)$ não significativo. Os valores entre parênteses referem-se ao erro-padrão; os entre colchetes, aos valores tabelados da estatística $\Phi$ e $\Phi^{\mathrm{m}}$ (TAR e M-TAR, respectivamente) e os entre chaves, ao p-valor obtido para o teste F.

Fonte: Elaborada pelos autores a partir dos dados da pesquisa.

O parâmetro threshold do modelo TAR $(\hat{\tau})$, estimado para cada par de mercados, permitiu verificar a assimetria na transmissão de preços. Observou-se que choques inferiores a 0,03\% no preço médio de Minas Gerais, por exemplo, são eliminados numa velocidade diferente da apresentada por choques maiores que esse percentual; em mercados mais distantes, como Santa Catarina e Rio Grande do Sul, esse percentual foi maior ( $0,37 \%$ e $0,45 \%$, em valor absoluto). As respectivas velocidades são captadas pelos valores de $\rho_{1}$ e $\rho_{2}$.

Os resultados indicaram, para todos os estados analisados, que os desvios superiores ao threshold foram eliminados ao longo do tempo. Todavia, quando os choques foram de baixa magnitude, ou seja, abaixo do threshold, não houve transmissão de preços entre São Paulo e Rio de Janeiro, Mato Grosso, Goiás e Santa Catarina, uma vez que a estimativa de $\rho_{2}$ não foi estatisticamente significativa. Nesses casos, pode-se afirmar que os custos de ajuste seriam superiores aos ganhos. 
A partir dos resultados do modelo M-TAR, foi possível identificar diferenças no ajustamento a choques de preços positivos e negativos no mercado central. As estimativas do parâmetro threshold foram relativamente maiores que as do TAR. No caso de Santa Catarina, por exemplo, aumentos de preço, superiores a 3,76\% do preço médio no estado, foram eliminados mais rapidamente que quedas nos preços, uma vez que $\left|\rho_{1}\right|>\left|\rho_{2}\right|$. A análise da significância dos parâmetros estimados mostrou que não houve integração de Minas Gerais, Espírito Santo e Mato Grosso com São Paulo, para desvios inferiores ao threshold, já que $\rho_{2}$ foi não significativo. Nos demais estados, à exceção de Rio de Janeiro, o resultado foi semelhante ao de Santa Catarina, ou seja, choques positivos, acima do threshold, foram eliminados mais rapidamente que choques negativos. Novamente, estados mais distantes do mercado central tiveram threshold mais elevados.

No Rio Grande do Sul, conforme já apresentado anteriormente, a transmissão de preços não ocorreu. Esse resultado diverge do de Pereira (2005), que utilizou o modelo de cointegração tradicional, e certamente está relacionado com a distância do estado em relação a São Paulo, que aumenta os custos de transação, principalmente os relacionados com frete. Além disso, conforme Urso (2007), no referido estado há indícios de uma formação de preços própria, uma vez que as condições do rebanho, da produção e de exportação são diferentes em relação ao restante do país.

Deve-se mencionar que, embora tenham sido identificadas assimetrias na transmissão de preços, os baixos valores do threshold sugerem que, em geral, não há grandes impedimentos à integração dos mercados. Esse resultado difere do que foi obtido por Mattos e Lima (2006), que analisaram a integração dos mercados de boi gordo de Minas Gerais e São Paulo, no período de 1972 a 2005, e o parâmetro threshold estimado foi de 0,097.

Pode-se justificar essa diferença pelas alterações na estrutura de produção do setor nos últimos anos. Conforme já discutido na Introdução deste estudo, os frigoríficos, visando reduzir os custos de compra dos animais, têm estabelecido unidades produtivas próximas às regiões produtoras. Dessa forma, reduzem custos relacionados com transportes, seguros, impostos e até mesmo com a coleta de informações. O baixo valor do threshold então faz sentido, já que neste trabalho foi considerado um período mais recente (1994 a 2008), diferentemente de Mattos e Lima (2006), cuja análise abrangeu as décadas de 1970 e 1980, nas quais as alterações no setor ainda não haviam ocorrido.

A proximidade entre frigoríficos e pecuaristas também pode ser apontada como explicação para o caso do Mato Grosso do Sul, no qual a especificação linear foi a mais adequada. Além de ter o terceiro maior rebanho bovino brasileiro, segundo dados do ANUALPEC (2008), o estado está entre os que possuem o maior número de frigoríficos do país. Além disso, o 
fato de grande parcela da carne produzida no estado ter São Paulo como destino, aumenta a possibilidade de transmissão simétrica de preços entre os dois mercados.

\section{Considerações Finais}

Dada a importância dos custos de transação num contexto de integração de mercados, e o fato de que as análises tradicionais não os levam em consideração, este estudo procurou analisar o efeito de tais custos sobre a integração do mercado brasileiro de boi gordo. Foram utilizados modelos TAR e M-TAR, versão modificada do teste de cointegração de Engle e Granger, que possibilita considerar assimetrias na transmissão de choques de preços entre os mercados.

Os resultados indicaram que os mercados eram integrados e foi confirmada presença de assimetrias na transmissão de preços. Encontraram-se evidências de que choques negativos e/ou de baixa magnitude foram eliminados de maneira distinta dos positivos. Em alguns casos, devido à não significância dos parâmetros estimados, não houve transmissão de preços. Nesses casos, certamente os benefícios advindos de alterações nos preços seriam menores que os custos de ajuste. Pôde-se concluir, portanto, que os custos de transação influenciaram a ligação espacial entre os mercados.

Não obstante, o efeito dos custos de transação sobre a integração dos mercados foi baixo, resultado que se deve às mudanças pelas quais vêm passando o setor, caracterizadas principalmente pela instalação de frigoríficos próximos às regiões produtoras. Com esse expediente, as firmas procuram reduzir os altos custos associados ao transporte de animais.

Por fim, sugere-se para trabalhos posteriores a utilização de modelos de correção de erro vetorial com threshold (TVEC) que contenham mais de um parâmetro threshold e múltiplos regimes de ajustamento.

\section{Referências}

ANUALPEC 2008. Anuário estatístico da pecuária de corte. São Paulo: FNP Consultoria e Comércio, 2008.

CHAN, K. S. Consistency and limiting distribution of the least squares estimator of a threshold autoregressive model. The Annals of Statistics, v. 21, n.1, p. 520-533, 1993.

CONSELHO NACIONAL DA PECUÁRIA DE CORTE. Balanço da pecuária bovídea de corte: 1994 a 2007. Disponível em: <http://www.cnpc.org.br/arquivos/Balanco.xls > . Acesso em: 11 nov. 2008.

DICKEY, D. A.; FULLER, W. A. Distribution of estimates for autoregressive time series with unit root. Journal of the American Statistics Association, v. 74, n.366, p. 427-431, 1979.

ENDERS, W. Applied econometric time series. New York: John Wiley, 1995. 433 p. 
ENDERS, W.; GRANGER, C. W. J. Unit-root tests and asymmetric adjustment with an example using the term structure of interest rates. Journal of Business and Economic Statistics, v. 16, n.3, p. 304-311, 1998.

ENDERS, W.; SIKLOS, P. Cointegration and threshold adjustment. Journal of Business and Economics Statistics, v. 19, n.2, p. 166-176, 2001.

ENGLE, R. F.; GRANGER, C. W. J. Cointegration and error correction: representation, estimation and testing. Econometrica, v. 35, n.1, p. 251-276. 1987.

EWING, B. T.; HAMMOUDEH, S. M.; THOMPSON, M. A.; Examining asymmetric behavior in US Petroleum futures and spot prices. The Energy Journal, v. 27, n.3, p. 9-23, 2006.

FACKLER, P. L.; GOODWIN, B. K. Spatial price analysis. In: RAUSSER, G. C.; GARDEN, B. L. (Ed.). Handbook of agricultural economics. Amsterdam: North-Holland Press, 2001. p. 971-1024.

FAMINOW, M. D.; BENSON, B. L. Integration of spatial markets. American Journal of Agricultural Economics, v. 72, n.1, p. 49-62, 1990.

FRANSES, P. H.; HOBIJN, B. Critical values for unit root tests in seasonal time series. Journal of Applied Statistics, v. 24, n.1, p. 25-46, 1997.

GOLETTI, F.; CHRISTINA-TSIGAS, E. Analyzing market integration. In: SCOTTI, G. J. (Ed.). Prices, products and people: analyzing agricultural markets in developing countries. Boulder: Lynne Riener, 1995.

GONZÁLEZ-RIVERA, G.; HELFAND, S. M. Desenvolvimento econômico e os determinantes da integração espacial nos mercados agrícolas. In: HELFAND, S. M.; REZENDE, G. C. (Ed.). Região e espaço no desenvolvimento agrícola brasileiro. Rio de Janeiro: IPEA, 2003. p. 121-147.

. The extent, pattern and degree of market integration: a multivariate approach for the Brazilian rice market. American Journal of Agricultural Economics, v. 83, n.3, p. 576-592, 2001.

GOODWIN, B. K.; HOLT, M. T. Price transmission and asymmetric adjustment in the U.S. beef sector. American Journal of Agricultural Economics, v. 81, n.3, p. 630-637, 1999.

GOODWIN, B. K.; PIGGOTT, N. E. Spatial market integration in the presence of threshold effects. American Journal of Agricultural Economics, v. 83, n.2, p. 302-317, 2001.

JOHANSEN, S. Statistical analysis of cointegration vectors. Journal of Economics Dynamics E Control, v. 12, n.2-3, p. 231-254, 1988.

LO, M.C.; ZIVOT, E. Threshold cointegration and nonlinear adjustment to the law of one price. Macroeconomic Dynamics, v. 5, n.4, p. 533-576, 2001.

MACKINNON, J. G. Critical values for cointegration tests. In: ENGLE, R. F., GRANGER, C. W. J. (Ed.), Long-run Economic Relationships. Oxford: Oxford University Press, 1991.

MADALLA, G. S.; KIM, I. M. Unit roots, cointegration, and structural change. Cambridge: Cambridge University Press, 1998. 505p.

MATTOS, L. B. Efeitos de custos de transação sobre a Integração espacial de mercados regionais de carne de frango no Brasil. 162 p. 2008. Tese (Doutorado em Economia Aplicada) - Universidade Federal de Viçosa, 2008. 
MATTOS, L. B.; LIMA, J. E. Integração espacial do mercado de boi gordo entre os estados de Minas Gerais e São Paulo na presença do efeito threshold. In: CONGRESSO DA SOCIEDADE BRASILEIRA DE ECONOMIA E SOCIOLOGIA RURAL, 44., 2006, Fortaleza. Anais... Brasília: SOBER, 2006.

OBSTFELD, M.; TAYLOR, A. M. Nonlinear aspects of goods-market arbitrage and adjustment: heckscher's commodity points revisited. Journal of the Japanese and International Economies, v. 11, n.4, p. 441-479, 1997.

PEREIRA, L. R. R. Integração espacial no mercado brasileiro de boi gordo. 166 p. 2005. Tese (Doutorado em Economia Aplicada) - Universidade Federal de Viçosa, 2005.

URSO, F. S. P. A cadeia da carne bovina no Brasil: uma análise de poder de mercado e teoria da informação. 113 p. 2007. Tese (Doutorado em Economia de Empresas) - Fundação Getúlio Vargas, 2007.

WANE, A.; GILBERT, S.; DIBOOGLU, S. Critical values of the empirical F-distribution for threshold autoregressive and momentum threshold models. Discussion Paper 2004-13: Department of Economics Southern Illinois University, 2004.

ZEN, S. Integração entre os mercados de boi gordo e de carne bovina nas regiões centro-oeste e sudeste do Brasil. 81 p. 1997. Dissertação (Mestrado em Economia Aplicada) - Escola Superior de Agricultura "Luiz de Queiroz", 1997.

Recebido em: 25/03/2009. Aceito em: 25/08/2009. 